\title{
Solving the Agricultural Land Allocation Problem by Constraint-Based Local Search
}

\author{
Quoc Trung BUI ${ }^{1}$, Quang Dung PHAM ${ }^{2}$, and Yves Deville ${ }^{1}$ \\ 1 ICTEAM, Université catholique de Louvain, Belgium \\ \{quoc.bui, Yves.Deville\}@uclouvain.be \\ 2 SoICT, Hanoi University of Science and Technology, Vietnam \\ dungpqesoict.hut.edu.vn
}

\begin{abstract}
Agricultural land allocation is a problem that exists in most provinces in Vietnam. Each household owns many disconnected parcels, which reduces agricultural development. The solution to the problem is to repartition this agricutural land among the households, while satisfying some criteria. Historically, this problem has been approached neither using optimization technology nor computer science. The present paper describes the formulation of the problem and proposes a constraint-based local search algorithm for solving it. Experimental results on real data in Dong Trung village show that the solution computed by our algorithm is better than traditional solutions.
\end{abstract}

\section{Introduction}

In most provinces of Vietnam, agricultural land is still fragmented. One household has many parcels of different land categories (each category corresponds to a certain quality) from different fields. These parcels are very small and scattered. For example, in Vinh Phuc province, one household might have 47 parcels, each of which has the area of about ten square meters. The fact that each households has many separated small parcels leads to a lot of difficulties. First, households can not use machines for cultivating their small parcels. Second, fragmented parcels require a very high cost for visiting and controlling them. Third, the excessive number of tracks between the parcels results in a waste of agricultural land. Finally, projects of agricultural development are confronted with many difficulties caused by the huge number of small parcels.

The Vietnamese government promulgated a policy to overcomes this limitation. This consists of merging small parcels into large fields and then repartitioning these fields into larger parcels. In provinces where the policy was carried out, the results obtained have been very promising. After merging and repartitioning, the number of parcels held by a household markedly decreases (e.g., Bac Ninh [1], a reduction by a factor of 10) and the area of each parcel increases, with the rice output increasing considerably (e.g., Quang Nam [5], an increase of 20\%-25\%). Today, this land reallocation process has only been applied in few vietnamese provinces.

After merging the existing parcels (specified in the past), we have a set of fields of different categories (there might be several fields of the same category) and a set of households; each household has an expected area of agricultural land for each category. We need to specify, for each household, the parcel (the area and the position of the parcel) in each field to avoid the above limitation. We consider fields of various shapes, i.e., rectangle, trapezium, quadrangle. But, for ease of presentation of the problem, we consider rectangular fields. Our solution however handles any quadrangular shape. We 
first present the traditional solution that people used in the past for allocating parcels to households, which does not use either computer science or optimization technology.

Traditional solution The Vietnamese government promulgated the following instructions to guide farmers in repartitioning the agricultural land. Fields are classified into categories (1-4) according to types of land quality, and determining a system of coefficients that presents an equivalence between categories. For example, a system of coefficients for three land categories $\langle 1.0,1.2,1.4\rangle$ in which $1 \mathrm{~m}^{2}$ of the first land category is equivalent to $1.2 \mathrm{~m}^{2}$ of the second land category and $1.4 \mathrm{~m}^{2}$ of the third land category. Fields of each land category are considered in turn for division into parcels, with the following rules:

1. The order of the fields is determined based on their land categories and geometrical positions and this is decided by the authorities.

2. The order of households is also determined by lot and households are assigned parcels with respect to this order. Suppose that this sorted list is $h_{1}, \ldots, h_{n}$.

3. Each field is divided into zones $z_{1}, \ldots, z_{k}$ of width $40-50$ meters by lines which are parallel to one side of the field.

4. Each zone $z_{i}$ is iteratively divided into parcels corresponding to a sequence of households $h_{j}, h_{j+1}, \ldots, h_{p}$ by parallel lines that are perpendicular to the parallel lines already used to separate the field into the zones. The next zone $z_{i+1}$ will then be partitioned into parcels for households $h_{p+1}, \ldots$

5. At each step, suppose that household $h_{i}$ under consideration, the current zone is $z_{j}$, and the current land category is $c$. The remaining area of $z_{j}$ is $R \mathrm{~m}^{2}$. If $R$ is greater than or equal to the expected area of $h_{i}$, then the next parcel of $z_{j}$ will be allocated to $h_{i}$. Otherwise, $h_{i}$ needs a supplementary area of $S \mathrm{~m}^{2}$. The following situations may occur:

- $R$ is smaller than 100 , in which case the previous household $h_{i-1}$ will receive this remaining area of $R \mathrm{~m}^{2}$. An equivalent (to $R \mathrm{~m}^{2}$ of category $c$ ) area of the next category $c+1$ will be subtracted from the expected area of $h_{i-1}$ for the category $c+1$. The next zone $z_{j+1}$ will then be considered for allocation to $h_{i}$.

- Both $R$ and $S$ are greater than or equal to 100 . The household $h_{i}$ is allocated this remaining area of $z_{j}\left(R \mathrm{~m}^{2}\right)$, and will then receive a parcel of $S \mathrm{~m}^{2}$ in the next considered zone or field.

- $R$ is greater than or equal to 100 , but $S$ is less than 100 . The household $h_{i}$ is allocated this remaining area of $R \mathrm{~m}^{2}$ and an equivalent (to $S \mathrm{~m}^{2}$ of category c) area of the next category $c+1$ will be added to the expected area of $h_{i}$ for the category $c+1$.

Limitations of the traditional solution First, a lot of households have allocated areas which are different from their expected areas. The cause of this comes mainly from dividing a field into zones of fixed width, $40-50 \mathrm{~m}$. Then, the threshold $100 \mathrm{~m}^{2}$ may be not suitable for provinces where each household has thousands of square meters of agricultural land. In that case, there may be small parcels (with areas slightly more than $100 \mathrm{~m}^{2}$ ) next to large parcels (with areas of some thousands of square meters). Finally, the instructions of the government do not take into account other expectations of the farmers, such as optimizing the number of parcels next to the canals, minimizing the distance from the house to the parcel, a nice form of the parcels, etc. 
Fig. 1: A solution guided by the instructions of the government with five households $H 1, H 2, H 3, H 4$, and $H 5$, and two fields of two different land categories with coefficients $\langle 1,1\rangle$. The expected areas of the first land category of the five households are $300,400,250,300$, and $250 \mathrm{~m}^{2}$ (left part of the figure) and 250, 260, 560, 250, and $180 \mathrm{~m}^{2}$ of the second land category (right part of the figure). Applying the instructions of the government, each field is separated into three zones of width $50 \mathrm{~m}$, and the allocated areas of the households are $300,400,300,300$, and $200 \mathrm{~m}^{2}$ in the field on the left; and $250,250,500,250$, and $250 \mathrm{~m}^{2}$ in the field on the right. In this solution, household $\mathrm{H}_{2}$ has three parcels of two land categories and households $H 3, H 4, H 5$ are allocated parcels whose areas are different from their corresponding expected areas.

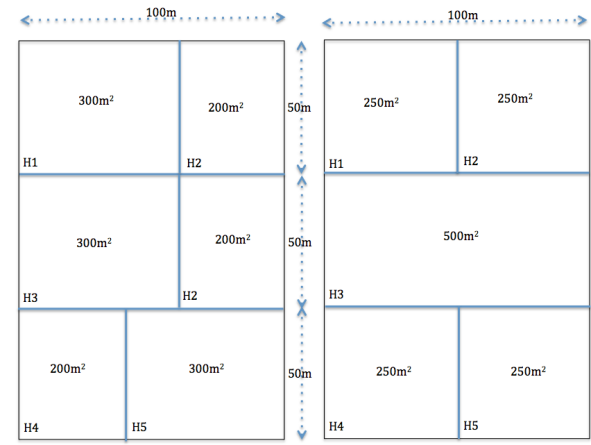

Objective of the present paper The objective of the present paper is to improve on the traditional solution to the agricultural land allocation (ALA) problem by applying optimization technology. We hope that the developped method could be used in the forthcoming land reallocation in Vietnam. We propose a constraint-based local search algorithm for solving this problem. A local search algorithm typically starts from a solution and moves from one solution to a neighboring solution in the hope of improving an objective function that guides the search. A constraint-based local search algorithm is a local search algorithm that uses the violation of constraints and the evaluation of objective functions to guide the search [8]. ALA consists of two successive subproblems:

- PArea is the problem of computing the area of the parcel allocated to each household in each field. We do not consider any order between households.

- PPos is the problem of specifying the exact positions of the parcels allocated to the households. From the solution to PArea, we know, for each field, the set of households and their allocated areas from this field, and we have to determine the exact positions of the parcels for these households. This problem has been considered and solved in [3] but only for those cases in which the given field has the shape of a rectangle, triangle, or trapezium. We propose in this paper an approach for partitioning the fields with general quadrangular shape, which appear frequently in reality.

Contribution We describe the problem formulation and a constraint-based local search algorithm for solving the ALA problem. We solve the PArea subproblem. For the PPos subproblem, we extend the results in [3] to partition a quadrangular field into parcels. We test the proposed algorithm on real data. The experimental results show the efficiency of the proposed algorithm compared with the traditional solution.

\section{PArea: Computing the Parcel Areas}

Input (1) A set of land categories $\mathcal{C}=\{1, \ldots, m\}$ with a system of coefficients $\left(\alpha_{1}, \ldots, \alpha_{m}\right)$ that present the equivalences between the land categories. (2) A set of fields $\mathcal{F}=\{1, \ldots, p\}$, each field $f \in \mathcal{F}$ is associated with an area $A(f)$ and a land category $C(f) \in \mathcal{C}$. (3) A set of households $\mathcal{H}=\{1, \ldots, n\}$, each household $h \in \mathcal{H}$ is associated with:

- a vector of expected areas $E A(h)=(A(h, 1), \ldots, A(h, m))$, in which $A(h, c)(h \in \mathcal{H}, c \in \mathcal{C})$ is the expected area of land category $c$. 
- a vector of distances $(d(h, 1), \ldots, d(h, p))$, in which $d(h, f)(h \in \mathcal{H}, f \in \mathcal{F})$ is the distance from the house of household $h$ to the center of field $f$.

Note that the equation $\forall c \in \mathcal{C}, \sum_{f \in \mathcal{F}: C(f)=c} A(f)=\sum_{h \in \mathcal{H}} A(h, c)$ is always ensured. Output The output is the allocated area of each household in each field.

Ideally, we desire to have a solution in which each household receives a unique parcel with the corresponding expected area in some field for each land category if the corresponding expected area is greater than zero. Unfortunately, this often cannot be arranged. For example, if we have two fields of areas $300 \mathrm{~m}^{2}$ and $700 \mathrm{~m}^{2}$ of category 1 , we have four households whose expected areas of land category 1 are respectively $200 \mathrm{~m}^{2}, 200 \mathrm{~m}^{2}, 400 \mathrm{~m}^{2}$, and $200 \mathrm{~m}^{2}$. In our approach, we prioritize the solutions in which each household receives a unique parcel in some field for each land category if the households expected area of this category is greater than zero. Otherwise, this household receives no parcel. In addition, we accept solutions in which some households have allocated areas different from their expected areas, and we try to minimize this difference.

In our approach, we iteratively allocate parcels to households for each land category, starting with land category 1 and finishing with land category $m$. The results computed for each land category $c$ may change the input of the expected areas of the households for the next land category:

- For a household who receives an additional area $a$ out of its expected area of land category $c$, its expected area for the land category $c+1$ will be lessened by $a \times \frac{\alpha_{c+1}}{\alpha_{c}}$

- For a household who receives area $a$ less than its expected area of land category $c$, its expected area for land category $c+1$ will be increased by $a \times \frac{\alpha_{c+1}}{\alpha_{c}}$

In the following, we give a mathematical formulation of the problem PArea for a specific land category $c$.

Input (1) A set of fields $\mathcal{F}_{c}=\left\{f_{1}, f_{2}, \ldots, f_{q}\right\} \subseteq \mathcal{F}$ of land category $c$. (2) A set of households whose expected areas of land category $c$ are greater than zero $\mathcal{H}_{c}=$ $\left\{h_{1}, \ldots, h_{k}\right\} \subseteq \mathcal{H}$

Decision variables Variable $a(h, f)\left(h \in \mathcal{H}_{c}, f \in \mathcal{F}_{c}\right)$ presents the allocated area of household $h$ in field $f$.

Invariant $F(h)=\left\{f \in \mathcal{F}_{c} \mid a(h, f)>0\right\}, \forall h \in \mathcal{H}_{c}$ represents the set of fields of land category $c$ where the household $h$ is allocated non-zero areas.

Constraints C1: $A(f)=\sum_{h \in \mathcal{H}_{c}} a(h, f), \forall f \in \mathcal{F}_{c}$. C2: $|F(h)|=1, \forall h \in \mathcal{H}_{c}$. Constraint $\mathbf{C 1}$ states that the sum of areas allocated to households in each field is equal to the area of that field, and $\mathbf{C 2}$ specifies that each household receives a unique parcel in some field.

Objectives In order to respond to the expectations of the farmers, we propose the two following objectives, which are to be minimized: $F_{0}(c)=$ $\sum_{h \in \mathcal{H}_{c}, f \in \mathcal{F}_{c}: a(h, f)>0} d(h, f)$ and $F_{1}=\sum_{h \in \mathcal{H}_{c}}\left|\sum_{f \in \mathcal{F}_{c}} a(h, f)-A(h, c)\right|$, in which $F_{0}(c)$ is the sum of distances from the houses of the households to the centers of the fields in which their allocated parcels are located, and $F_{1}$ is the sum of the differences between the allocated areas and the expected areas of the households.

A constraint-based local search algorithm has been developped for solving problem PArea for each land category $c$. The algorithm uses tabu lists in order to avoid revisiting 
solutions already explored. We always ensure the constraint $\mathbf{C 2}$ holds by modeling a solution by a $k$-dimensional vector $\left(x\left(h_{1}\right), \ldots, x\left(h_{k}\right)\right)$ in which $x\left(h_{i}\right) \in \mathcal{F}_{c}\left(h_{i} \in \mathcal{H}_{c}\right)$ indicates the field wherein the household $h_{i}$ is allocated a parcel. During the search, there are invariants $\Delta(f)=\left|A(f)-\sum_{h_{i} \in \mathcal{H}_{c}}\left(x\left(h_{i}\right)=f\right) * A\left(h_{i}, c\right)\right|$ representing the difference between the area of the field $f$ and the sum of the expected areas of the households allocated in this field. Our algorithm will find solutions minimizing $\sum_{f \in \mathcal{F}_{c}} \Delta(f)$ and $F_{0}$. Finally, the actually allocated areas of the households will be adapted based on the solution computed in order to satisfy the constraint $\mathbf{C 1}$.

The algorithm proceeds in two steps. Step 1 determines for each field of land category $c$ a group of households who have parcels in this field. The solution is represented by a vector $S=\left(x\left(h_{1}\right), \ldots, x\left(h_{k}\right)\right)$ in which $x\left(h_{i}\right) \in \mathcal{F}_{c}$ is a field of land category $c$ in which a parcel allocated to household $h_{i}$ is located, $\forall h_{i} \in \mathcal{H}_{c}$. Our algorithm considers two neighborhoods:

1. Change-based neighborhood

$$
N_{1}(S)=\left\{\left(x\left(h_{1}\right), \ldots, x^{\prime}\left(h_{i}\right), \ldots, x\left(h_{k}\right)\right) \mid h_{i} \in \mathcal{H}_{c}, x\left(h_{i}\right) \neq x^{\prime}\left(h_{i}\right) \in \mathcal{F}_{c}\right\}
$$

2. Swap-based neighborhood $N_{2}(S)=\left\{\left(x\left(h_{1}\right), \ldots, x\left(h_{i+j}\right), \ldots, x\left(h_{i}\right), \ldots x\left(h_{k}\right)\right) \mid\right.$ $\left.x\left(h_{i}\right) \neq x\left(h_{i+j}\right) \in \mathcal{F}_{c}, 1 \leq i<i+j \leq k\right\}$

In step 1, we suppose each household is allocated a quantity of area which is equal to its expected area. Due to this assumption, there exist, for each field, a difference $\delta$ between the area of the field and the sum of the allocated areas in this field. The goal of step 2 of our algorithm is to adapt the allocated area such that the sum of the allocated areas in the field fits the area of that field.

\section{PPos: Finding the Positions of the Parcels}

When problem PArea has been solved, we have, for each household, their allocated area in each field. The overall goal of the solution of PPos is to determine the exact position of the parcels in each field. In our previous paper [3], we specified the problem and proposed tabu search algorithms for PPos when the shape of fields was restricted to be a triangle, a trapezium, or a rectangle.

A field is a large quadrangle where hundreds of households must be assigned a parcel. The field will be decomposed using horizontal and vertical lines as illustrated in Figure 2 . The field will be partitioned into zones by horizontal lines. Each zone is then partitioned into parcels by vertical lines which are allocated to households. Each household will be assigned only one parcel in the field. When one or more sides of the quadrangle are close to a canal, one has to maximize the number of parcels close to the canal (see Figure 2) as this is an important issue for Vietnamese farmers. The PPos problem is then divided into two independent subproblems.

- Problem P1: The given field has one or more canals that are located on its sides. This problem computes the zones and parcels next to the canals that are assigned to the househoulds.

- Problem P2: The remainder of the field, obtained by removing the zones computed by $\mathbf{P 1}$, has no canal on its sides. This remaining area, considered as a new field, is then partitioned into zones and parcels assigned to households.

The problem $\mathbf{P 1}$ is viewed as a subset selection problem: we need to find a subset of households, satisfying some constraints while optimizing some objective function. The 
constraints are that each parcel must fit the exact area corresponding to a household, and that the ratio between the height and the width of a parcel must be bounded by some given constant in order to have parcels with an efficient shape. The objective function is to maximize the number of parcels next to the canal. A second, less important objective, is to have parcels with a shape close to a square. A tabu search is described in [3], where three neighborhoods are considered: removal-based, insertion-based, and swap-based neighborhoods.

The problem $\mathbf{P 2}$ is viewed as a set partitioning problem. Given a set of households, we need to partition this set into subsets of households, one subset corresponding to one zone of the field. Each zone is then easily decomposed into parcels. Each parcel will thus fit the exact area corresponding to a household. The objective is to have parcels with a shape close to a square. A tabu search is described in [3], where two neighborhoods are considered: move-based and swap-based neighborhoods.

In [3], we restricted the shape of the field to be that of a triangle, trapezium, or a rectangle. However, most fields in reality are quadrangular. We here extend the problem to quadrangular fields. The idea is that a quadrangular field can be decomposed into smaller fields or can be approximated by a field of the shape of a trapezium, so that we can directly reuse the tabu search algorithms in [3].

When the quadrangular field has a shape close to a trapezium, the field is approximated by a trapezium with the same area. On the top of Figure 2, a quadrangle $A B C D$ (field of quadrangle $A B C D$ for short) is approximated by a trapezium $A B^{\prime} C^{\prime} D$, then the algorithms in [3] are applied to partition the trapezium $A B^{\prime} C^{\prime} D$ into the zones $B^{\prime} E_{1} F_{1} C^{\prime}$, $E_{1} F_{1} F_{2} E_{2}, E_{2} F_{2} F_{3} E_{3}$, and $E_{3} F_{3} D A$ is divided into parcels.

When the quadrangle is not close to a trapezium, the field is decomposed into two parts: a triangular field and a pentagonal field that is approximated by a field with the shape of a trapezium with the same area. On the bottom of Figure 2, a quadrangle $A B C D$ is separated into a triangle $C H G$ and a pentagon $A B H G D$. Then the pentagon $A B H G D$ is approximated by a trapezium. The algorithms in [3] can then be applied to partition these two fields into zones and parcels.

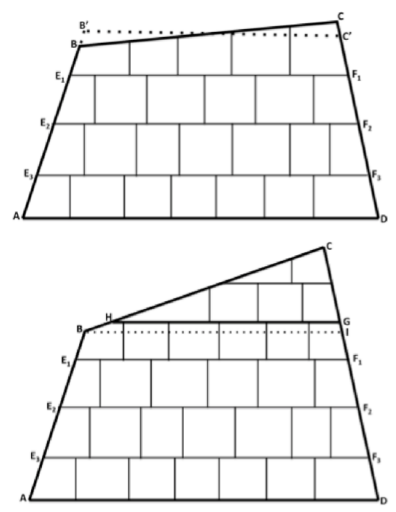

Illustration of two ways of separation
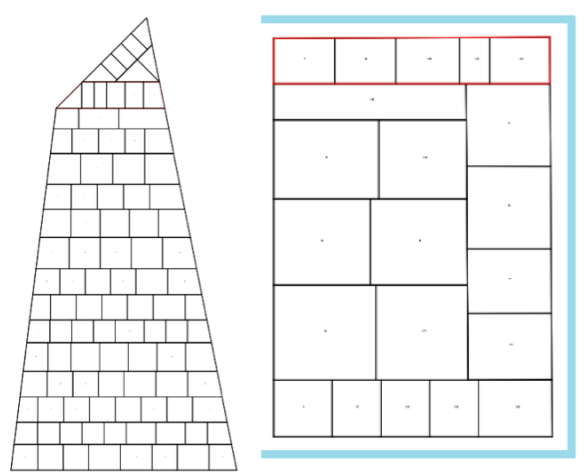

Example of partitioning quadrangular fields into parcels

Fig. 2: Illustration and Example of partitioning quadrangular fileds 
In the above decomposition of a quadrangular field into a pentagon and a triangle, we need to decide the position of the cut (line $H G$ in Figure 2). A constraint is that the triangular field ( $\mathrm{CHG}$ in Figure 2) should have an area corresponding to a subset of so that the sum of the allocated areas of a group of households. To identify this cut, we solve a subset selection problem [7] as follows: given a set $P=\left\{a_{1}, \ldots, a_{p}\right\}, P \subset \mathbb{R}^{+}$ and a threshold $\mathcal{T} \in \mathbb{R}^{+}$, the overall goal is to determine a subset $S P \subseteq P$ satisfying the constraint $\mathcal{T} \geq \sum_{a \in S P} a$ and minimizing the objective function $\left(\mathcal{T}-\sum_{a \in S P} a\right)$. In this problem, the threshold $\mathcal{T}$ is the maximum area of the triangular shape (in Figure 2), $\mathcal{T}$ is the area of the triangle $B C I$ ). To solve this subset problem, we developed a simple tabu search algorithm using the two neighborhoods of a solution $S P \subseteq P$ : Changebased neighborhood $N_{1}(S P)=\{S P \cup a \mid a \notin S P, a \in P\} \cup\{S P \backslash\{a\} \mid a \in S P\}$ and Swap-based neighborhood $N_{2}(S P)=\{S P \cup a \backslash\{b\} \mid a \in P, a \notin S P, b \in S P\}$.

\section{Experiments}

We selected the $10^{t h}$ hamlet of Dong Trung village, Tien Hai district, Thai Binh province, Vietnam, to try our algorithms. We collected all the data before and after the land grouping of this hamlet. In this hamlet, there are 103 households, 3 land categories $\{c 1, c 2, c 3\}$, and 24 fields adding to $204,744 \mathrm{~m}^{2}$ ( 8 fields of $c 1,7$ fields of $c 2$ and 9 fields of $c 3$ ). In the $10^{\text {th }}$ hamlet, the agricultural land allocation problem was already carried out by using the instructions of the government. The system of coefficients was set to $\langle 1.0,1.0,1.0\rangle$ by the farmers. In the existing solution by the farmers guided by the instructions of the government, almost all households have three parcels, some households are allocated four parcels.

Our Tabu search algorithm was implemented in the COMET programming language [4] and always returns the best solution obtained in the search. The time limit for each execution was set to two minutes. The length of the tabu lists was set to 20 . The experiments were performed on XEN virtual machines with one core of a CPU Intel Core2 Quad Q6600 @2.40GHz and 1GB of RAM.

Figure 2 illustrates the partitioning of two quadrangular fields into parcels by our extended algorithm for solving PPos, in which the field on the left of the figure does not touch a water source and the field on the right of the figure is close to three canals (blue lines). We compared the solutions computed by the tabu search algorithms with the existing solution by the farmers. The criteria for evaluating the solutions are the differences between the expected area and the allocated area for each household, for each land category, and the total distances from the houses of the households to the centers of the fields where their parcels are located. In the traditional solution, there are households whose expected area is equal to their allocated area. However, for the other households, the differences between the expected areas and the allocated areas are very high. This shows a greater inequality between households. In our approach, the solution computed ensures greater equality between the households in the sense that all households can tolerate the resulting differences. Moreover, the differences produced by our solution are very small.

In figure 3, the last three columns present a summary of 20 executions of our tabu search algorithm. Column $M I N$ (resp. $A V G$ and $M A X$ ) presents the minimum (average and maximum) value of the 20 solutions. Other fairness criteria, such as in $[2,6]$, could also be used. 
Fig. 3: Comparing the traditional solution and our solution, where

$$
\begin{aligned}
& \operatorname{diff}(h, c)=A(h, c)-\sum_{f \in \mathcal{F}: C(f)=c} a(h, f) \\
& \operatorname{Max}(c)=\operatorname{Max}\{|\operatorname{diff}(h, c)| \mid h \in \mathcal{H}\} \\
& \operatorname{Avg}(c)=\frac{1}{\left|\mathcal{H}_{c}\right|} \sum_{h \in \mathcal{H}}|\operatorname{diff}(h, c)| \\
& \operatorname{Var}(c)=\sqrt{\frac{1}{\left|\mathcal{H}_{c}\right|} \sum_{h \in \mathcal{H}}(|\operatorname{diff}(h, c)|-\operatorname{Avg}(c))^{2}} \\
& \operatorname{Max}=\operatorname{Max}\left\{\left|\sum_{c \in \mathcal{C}} \operatorname{diff}(h, c)\right| \mid h \in \mathcal{H}\right\} \\
& \operatorname{Avg}=\frac{1}{\mathcal{H}} \sum_{h \in \mathcal{H}}\left|\sum_{c \in \mathcal{C}} \operatorname{diff}(h, c)\right| \\
& \operatorname{Var}=\sqrt{\frac{1}{\mathcal{H}} \sum_{h \in \mathcal{H}}\left(\left|\sum_{c \in \mathcal{C}} \operatorname{diff}(h, c)\right|-A v g\right)^{2}} \\
& F_{0}=\sum_{c \in \mathcal{C}} F_{0}(c)
\end{aligned}
$$

\begin{tabular}{|c|c|c|c|c|}
\hline \multirow[t]{2}{*}{ Criteria } & \multirow{2}{*}{$\begin{array}{c}\text { Trad. } \\
\text { sol. }\end{array}$} & \multicolumn{3}{|c|}{20 executions of tabu search } \\
\hline & & $M I N$ & $A V G$ & $M A X$ \\
\hline $\operatorname{Max}(c 1)$ & 88 & 5 & 7.75 & 15 \\
\hline $\operatorname{Avg}(c 1)$ & 39.51 & 2.06 & 2.39 & 3.21 \\
\hline $\operatorname{Var}(c 1)$ & 29.37 & 1.22 & 1.87 & 3.27 \\
\hline $\operatorname{Max}(c 2)$ & 98 & 6 & 8.45 & 17 \\
\hline $\operatorname{Avg}(c 2)$ & 32.93 & 1.95 & 2.39 & 3.16 \\
\hline $\operatorname{Var}(c 2)$ & 25.31 & 1.29 & 1.96 & 3.23 \\
\hline $\operatorname{Max}(c 3)$ & 89 & 1 & 3.35 & 6 \\
\hline$A v g(c 3)$ & 13.96 & 0.28 & 0.85 & 1.68 \\
\hline $\operatorname{Var}(c 3)$ & 17.73 & 0.45 & 0.90 & 1.43 \\
\hline $\operatorname{Max}$ & 89 & 1 & 1.15 & 2 \\
\hline$A v g$ & 13.65 & 0.04 & 0.17 & 0.33 \\
\hline Var & 16.92 & 0.19 & 0.36 & 0.51 \\
\hline Added parcels & 10 & 0 & 0 & 0 \\
\hline$F_{0}(\mathrm{~km})$ & 270.79 & 261.55 & 265.10 & 271.36 \\
\hline
\end{tabular}

Our solutions are clearly better than the existing solution obtained by the application of the instructions of the government. There does not exist any household that has more than two parcels. This means that our solutions always satisfy constraint C2. In the existing solution by the farmers, there are 10 households that have four parcels. The values of the first 12 criteria of our solutions are always much smaller than those for the existing solution by the farmers. The value of the objective function $F_{0}$ in most of our solutions is slightly smaller than that of the existing solution by the farmers.

This experiment has been presented to the Vice President of the Dong Trung village (Thai Binh province). This village is composed of 1460 households, and the land has already been reallocated using the traditional instructions of the government. The solutions produced by our algorithms were considered as a significant improvement over the traditional reallocation. The very low difference between the expected area and the allocated area for each household, the absence of added parcels and the form of parcels with a shape close to a square were particularly appreciated. His conclusion was that our tool should be used to the land allocation of the remaining Vietnamese districts and provinces.

\section{Conclusion}

In this paper, we solved the agricultural land allocation problem that has recently emerged in many provinces in Vietnam, using constraint-based local search algorithms. The problem comprises two subproblems. The first one is to compute the set of households that are allocated parcels in each agricutural field, and the second one is to specify the exact position of each parcel allocated in each agricutural field. For the first subproblem, we described its formulation and proposed a constraint-based local search algorithm for solving it. For the second subproblem, we extended the results in [3] to compute the position of each parcel allocated in a field with the shape of a generic quadrangle. The experimental results show that our approach yields better solutions than the traditional solution. The experimental results were also validated by a Vietnamese authority in land allocation.

We are now reimplementing the algorithms as a $\mathrm{C}++$ tool that could be used by the different vietnamese districts. This tool will also be extended to handle specific constraints and objective functions, to meet the expectations of the farmers in various provinces. Comparison with other optimization approches will also be investigated. 


\section{References}

1. Bac Ninh News. Hieu qua don dien doi thua o yen phong. www.bacninh.gov.vn, 2010.

2. S. Bouveret and M. Lemaître. Computing leximin-optimal solutions in constraint networks. Artif. Intell., 173(2):343-364, Feb. 2009.

3. Q. T. Bui, Q.-D. Pham, and Y. Deville. Constraint-based local search for fields partitioning problem. In Proceedings of the Second Symposium on Information and Communication Technology, SoICT '11, pages 19-28, New York, NY, USA, 2011. ACM.

4. D. D. T. Inc. Comet Tutorial, 2004.

5. Le Van. Hoan thanh don dien doi thua. Dan Viet, 2011.

6. W. Ogryczak and T. Sliwinski. On solving linear programs with the ordered weighted averaging objective. European Journal of Operational Research, 148(1):80-91, 2003.

7. K. Pruhs and G. J. Woeginger. Approximation schemes for a class of subset selection problems. Theor. Comput. Sci., 382(2):151-156, Aug. 2007.

8. P. Van Hentenryck and L. Michel. Constraint-based local search. The MIT Press, London, England, 2005. 